\title{
COVID-19 PANDEMIC: MISSED INITIAL AND FOLLOW UP APPOINTMENTS AT AN ACADEMIC MEDICAL INSTITUTION IN SOUTH INDIA
}

\author{
Presthiena E L Lofi ${ }^{1}$ \\ ${ }^{1}$ Jubilee Centre for Medical Research, \\ Jubilee Mission Medical College and \\ Research Institute, \\ Thrissur, Kerala, \\ India
}

\section{P R Varghese ${ }^{3}$}

${ }^{3}$ Jubilee Centre for Medical Research, Jubilee Mission Medical College and

Research Institute,

Thrissur, Kerala,

India

\author{
Indu K Gopi ${ }^{2}$ \\ ${ }^{2}$ Jubilee Centre for Medical Research, \\ Jubilee Mission Medical College and \\ Research Institute, \\ Thrissur, Kerala, \\ India
}

Corresponding Author

*Praveenlal Kuttichira, Prof of Psychiatry \& Principal, Jubilee Mission Medical College \& Research Institute, Thrissur, Kerala- 680005

Article DOI: https://doi.org/10.36713/epra6772

DOI No: 10.36713/epra6772

\begin{abstract}
The global outbreak of COVID-19 has greatly influenced the health care delivery system and health seeking behavior by patients. This study aimed to explore the missed patient appointments and the factors lead to missed appointments, at an academic medical institution in South India during COVID-19 pandemic. The study used descriptive retrospective survey design to collect register based data from all departments of the hospital during the period $1^{\text {st }}$ January 2020 to $30^{\text {th }}$ June 2020. Among the 8390 missed patient appointments reported during this period, 4670 patients were included in the sampling frame and 130 samples were selected using systematic sampling. Socio demographic variables, clinical variables and reasons for missed appointments were collected from hospital data base and telephonic interview. Age ranged from 1 month to 85 years with the median age of $42( \pm 22.9)$ years. Male female ratio was 1:1. Of this 69.5\% (90) patients were married and $41.5 \%$ was educated up to high school. Majority of the cases $(57.7 \%)$ belonged to nuclear family; highest representation was from the economic non priority subsidy. More than half of missed appointments were noted in the medicine department and the most of the appointments was review cases. Reported reasons for missed appointments were personal constraints (50.8\%), financial and family constraints (5.4\%) time constraints (5.4\%) hospital related reasons (4.6\%) and COVID-19 related reasons (2.3\%). During COVID-19 pandemic there was no significant number of missed appointments in the study centre. The missed appointment group belonged to non priority economic category. The majority of cases found were review appointments. When enquiries were marked departmental-wise for the missed appointments, medical departments had more missed appointments than others. The present study highlights the need for developing strategies to ensure healthy flow of non COVID health care services during pandemic period.
\end{abstract}

KEYWORDS: COVID-19, Health care services, Missed Appointments, Non-COVID Health Care, Pandemic 


\section{INTRODUCTION}

The COVID-19 is a global pandemic which have greatly influenced the health care seeking behaviour of the people. It adversely affected the routine health care delivery and health service uptake by patients. In this study, missed appointments are defined as hospital appointments scheduled by patients but did not attend. Considerable reduction in the number of patients seeking essential health care during the time of pandemic were reported during SARS, ${ }^{(1)} \mathrm{Ebola}^{(2)}$ and MERS-CoV.(3) One third of care seekers avoiding or delaying care seeking is reported from USA due to COVID-19.(4) A report from Spain showed a drop of $40 \%$ in the cardiac catheterization for STEMI.(5) A previous study from this centre on paradigm shift in health care seeking behavior of patients during $1^{\text {st }}$ January to $30^{\text {th }}$ June 2020 , identified $31.01 \%$ decrease in the number of patients seeking non COVID health care compared to the previous year.(6) Age wise fall percentage was highest in the pediatric group (37.22\%) and lowest in senior citizen group $(29.26 \%)$. There has been a significant drop in both in-patient and out-patient footfall for hospitals during this period.(7)(8)

It is critical to maintain essential non COVID health services especially for the vulnerable populations, such as children, older persons, people living with chronic conditions or disabilities.(9) Factors like distance from a service center and disease severity were reported to have a significant association with a reduction in the utilization of the health service delivery during pandemics.(5) However, impact of COVID 19 pandemic on careseeking behavior of the non-COVID patients is not well studied. Since this institution is in the epicenter of first COVID 19 case reported in the country, the caution and response of the non COVID patients have generated curiosity among public health workers.

\section{OBJECTIVES}

This study aimed to; (i) to identify the sociodemographic and clinical variables of the patients missed scheduled appointments for non-COVID health care during COVID-19 period (ii) to explore the factors lead the patients to missed appointments for non-covid health care during COVID-19 period

\section{METHODOLOGY}

Research Design: This study used descriptive retrospective survey design, based on data from the departments providing non COVID health care at an academic medical institution, from $1^{\text {st }}$ January 2020 to $30^{\text {th }}$ June 2020. Information about their appointments was extracted for this specific period and patients were filtered using the inclusion criteria: (1) patients with phone number in the booking register and (2) willing to participate in the study.
Study Population \& setting: Patients missed scheduled appointments for non COVID health care during COVID-19 period at an academic medical Institution in South India

Sampling and sample size: There were $1,89,140$ booked appointments in the hospital during this period, of which 19,965 were at emergency medicine dept. Excluding the cases at emergency medicine dept there were $1,69,175$ appointments. Among this 8390 missed appointments were reported during this period. Of this 4670 patients who met inclusion criteria formed the sampling frame. Sample size was calculated using Cochrane formula at 95\% confidence intervaland $3.5 \%$ precision. Sample sizes of 130 patients were selected using systematic sampling. By dividing the allocated sample size number to the total number of patients missed appointed during study period contributed the sampling interval. First case was selected using simple random sampling and every $35^{\text {th }}$ sample was selected using random sampling from the sampling frame and continued counting until the desired sample size was obtained.

Data collection: Demographic variables such as age, gender, marital status, illness, and department were collected from hospital register. The patients were approached over telephone and semi structured interview were conducted after getting their consent to collect data on education, economic activity, socioeconomic strata, family structure, and type of illness and factors of nonattendance. The patients were given with 6 point rating scale to mark their perception regarding general health and health in particular to the illness at the time of survey ( 0 - not alive, 1- very poor health, 2- poor health, 3- average health, 4- good health, 5- very good health). Data collected regarding the home remedies, self management measures used. The patients were also asked to report if they have consulted in the local health facility during the study period.

Data analysis: Microsoft Excel 2007 was used for data entry and management. Socio demographic data and clinical data analyzed in frequencies, percentage, median, and standard deviation using SPSS version 25 IBM. The factors lead to missed appointments were coded and content analysis done.

\section{RESULTS}

The proportion of the total booking cancellation to total appointments from the time period of $1^{\text {st }}$ January 2020 to $30^{\text {th }}$ June 2020 was $4.4 \%$. Among 130 samples selected, the age ranged from 1 month to 85 years with the median age of 42 $( \pm 22.9)$ years. Fifty five patients $(42.6 \%)$ who missed the appointments were in the age group of 3564 years. The missed appointment from the pediatric group was $20.2 \%$. There were $19.4 \%$ (15-34 years) and $18.5 \%$ ( $\geq 65$ years) missed appointments in the respective age groups. Out of 130 patients selected 
65 were males and 65 were females. Of this 90 $(69.5 \%)$ patients were married, $38(29.2 \%)$ were unmarried and $2(1.5 \%)$ were widowed. The education was up to high school in $41.5 \%$ and above high school in $33.8 \%$. The remaining 32 (23.1\%) were illiterates. Majority of the cases (57.7\%) belonged to nuclear family and $42.3 \%$ belonged to joint family.

Economically inactive patients were $71.1 \%$ and active patients were $28.9 \%$. The socio economic status of their family is also studied. The highest representation was from the non priority subsidy blue ration card holders $(31.4 \%)$ followed by priority households- Below poverty line $(28.9 \%)$, general non-priority $(26.4 \%)$, most economically backward $(2.5 \%)$, and no ration card $(2.5 \%)$. Among the total patients, those who attended departments except emergency were 160785 (89\%). Among them 48097 $(25.5 \%)$ were new cases and 112688 (59.9\%) were review cases. Out of 130 missed samples studied 87 $(66.9 \%)$ patients were old cases booked for review and $43(33.1 \%)$ were new cases.

Among the 130 missed samples studied, 13 $(10 \%)$ patients were booked for their cardiac complaints and $6(4.6 \%)$ patients with neurological complaints. The patients with respiratory, reproductive and surgical complaints were $3.8 \%$ in each (Fig 1). Among total samples studied 88(67.7\%) were booked for consultation in the medicine department, $18(13.8 \%)$ in the surgery department, $10(7.7 \%)$ in the pediatric department, $8(6.2 \%)$ in the obstetrics and gynecology departments, and 6(4.6\%) in the emergency medicine department (table 1).

Among 130 patients interviewed, 66 (50.8\%) patients reported personal constraints as reason for missed appointments, 7(5.4\%) patients reported financial and family constraints, 7(5.4\%) patients reported time constraints, $6(4.6 \%)$ patients reported hospital related reasons and $3(2.3 \%)$ persons reported COVID 19 related reasons. Among 130 samples $41(31.5 \%)$ patients not reported any reason for missed appointments (figure 2). The patients marked their perception on general health and health in particular to illness. Out of 130 samples, 80 (61.5\%) reported very good health status in particular to the disease and $81(61.8 \%)$ patients reported very good health in general as per their perception. Only four patients reported poor health both in particular to disease and general health. Three patients were not alive at the time of survey. Forty one (32\%) patients reported that they have managed with home remedies and $33(25.4 \%)$ continued with same prescription during this days of missed appointments. Only 7 $(5.3 \%)$ patients availed health care from local health facility.

\section{DISCUSSION}

The COVID-19 outbreak has profound impact in the delivery of essential health services in other areas of health care across the globe. ${ }^{(10)}$ Major impact has been found as the under-utilization of important health care services for patients with non-COVID-19 related health needs. As the pandemic continues it becomes more essential to find out different strategies to tackle these effects with minimal risk to the patients and health professionals. The inequities in health and health seeking behavior of people, arise because of the circumstances in which they grow, live, work, the systems put in place to deal with illness and their age. ${ }^{(11)}$

In the present study the middle aged category was the major proportion among the missed appointments. A previous study from this centre also reported $32 \%$ patient fall rate in the middle aged adult category during $1^{\text {st }}$ January to $30^{\text {th }}$ June 2020 when compared to the previous year. ${ }^{(6)}$ The studies from different parts of India reported that aging is a major risk factor for several debilitating and life threatening diseases ${ }^{(12)}$. Also non-adherence to long term treatment was found largely in the middle aged category. ${ }^{(13)}$ This draws attention to the reports that the chronic illness rates swell in the middle age. Hence it is a matter of importance to support middle aged group to manage their conditions by timely interventions.

Family is a crucial demographic variable when considering the health related outcomes. The studies conducted in health and medication adherence in relation to family support, reported both supportive and obstructive behavior from the family. In the present study majority of the patients missed appointment were married and belong to nuclear family. Findings from other studies also endorse interventions that help family members to develop actionable plans supporting patients' self-care and train them to communicate the health outcome. ${ }^{(14)}$

Education and socioeconomic status are two major factors influencing the way people live, their consequent chance of illness, and their risk of premature death. ${ }^{(15)}$ Previous investigations reported that low socioeconomic status was usually associated with increased risk of the disease conditions. ${ }^{(16)}$ In the present study a good proportion of patients missed scheduled appointments were educated and belonged to the non priority households. Hence it can be considered, education and the awareness thereby may lead them to strict adherence to the lockdown instructions and self management of the illness. As WHO reported, it is the right time for the governments, civil society, and other organizations to come together in taking action to improve the lives of citizens $\&$ achieving health equity. ${ }^{(11)}$

Evidences from studies conducted in the context of previous epidemics identified that people lose trust in the healthcare system and develop a fear of getting the disease from the health facility. Thus, they avoid seeking treatment for their illness or sending family members for medical care, the 
consequences of which may be increased mortality.(17) In our study the majority of patients missed appointments were follow up cases and the rate was high in the medical departments especially cardiac and neurology departments. A previous study from this centre also reported $31.4 \%$ fall rate in the review cases in the year 2020 when compared to 2019 with more decline in the medical departments. ${ }^{(6)}$

The total proportion of missed appointments to the total appointments during the study period was only $4.4 \%$. Most of patients reported that their perceived health was good in general and particular to the illness. The studies exploring the people's attitude towards COVID 19 Pandemic reported that the people are willing to follow government guidelines on quarantine and social distancing. ${ }^{(18)}$ The study results also showed that patients managed by following same prescriptions, home remedies, and self management instead of attending the appointments in hospital. Very few of them had to attend the local health care facility. The explored reasons for missed appointments were also personal in high percentage and very little in direct relation to COVID 19 pandemic. Hence it is clear that people inthis part of the country adhered to the COVID 19 protocols of government without a major change in health seeking behavior.

\section{CONCLUSION}

During COVID 19 pandemic there were no significant numbers of missed appointments in the study centre. The socio demographic data as identified in the study, the missed appointment group belonged to non- priority economic category. The majority of cases found were review appointments. When enquiries were marked departmental-wise for the missed appointments, medical departments had more missed appointments than others. The present study highlights the need for developing strategies to ensure healthy flow of non COVID health care services during pandemic period.

\section{ABBREVIATIONS}

COVID-19: Corona Virus Disease 19; SARS: Severe Acute Respiratory Syndrome; MERS-CoV: Middle East Respiratory Syndrome Corona Virus; STEMI: ST-Elevation Myocardial Infarction; WHO: World Health Organization

\section{Acknowledgement}

The authors thank Mr E V Vinu, Project

Management Officer, JMMC \& RI for providing help to filter data from hospital records and to Ms Mridula Vellore, Research Assistant ( Scientific writing) for editing the article.

Funding: Nil

\section{REFERENCES}

1. Aklilu, Tamirat M. et al. (2020), "The Impact of COVID-19 on Care Seeking Behavior of Patients at Tertiary Care Follow-up Clinics: A CrossSectional Telephone Survey. Addis Ababa, Ethiopia", medRxiv: 2020.11.25.20236224.

2. Chathukulam, Jos, and Joseph Tharamangalam. (2021), "The Kerala Model in the Time of COVID19: Rethinking State, Society and Democracy", World Development 137: 105207.

3. "Closing the Gap in a Generation: Health Equity through Action on the Social Determinants of Health - Final Report of the Commission on Social Determinants of Health." (February 16, 2021) https://www.who.int/publications-detailredirect/WHO-IER-CSDH-08.1.

4. Heiber, Michael, and W. Y. Wendy Lou. (2006), "Effect of the SARS Outbreak on Visits to a Community Hospital Emergency Department." CJEM 8(5), p.p: 323-28.

5. Hoenig, Kerstin, and Sebastian E. Wenz. (2020). "Education, Health Behavior, and Working Conditions during the Pandemic: Evidence from a German Sample", European Societies $O(O)$, p.p: $1-14$.

6. Iihara, N. et al. 2004. "Beliefs of Chronically Ill Japanese Patients That Lead to Intentional NonAdherence to Medication", Journal of Clinical Pharmacy and Therapeutics 29(5), p.p: 417-24.

7. Kezia Kuruvila, Praveenlal kuttichira, Varghese PR, Vinu EV, Indhu K Gopi (2020) "Paradigm Shift in Health Care Seeking Behavior: A Report from Central Kerala, India during COVID-19 Pandemic." CHRISMED J Health Res; 7:271-5.

8. Lee, K. D. et al. (2020), "Providing Essential Clinical Care for Non-COVID-19 Patients in a Seoul Metropolitan Acute Care Hospital amidst Ongoing Treatment of COVID-19 Patients.", , The Journal of Hospital Infection 106(4), p.p: 673-77.

9. Lee, Sun Young, Young Ho Khang, and Hwa Kyung Lim. (2019), "Impact of the 2015 Middle East Respiratory Syndrome Outbreak on Emergency Care Utilization and Mortality in South Korea.", Yonsei Medical Journal 60(8), p.p: 796-803.

10. Lewer, Dan et al. (2020). "Premature Mortality Attributable to Socioeconomic Inequality in England between 2003 and 2018: An Observational Study.” The Lancet Public Health 5(1), p.p: $33-41$.

11. Mayberry, Lindsay Satterwhite, and Chandra Y. Osborn. (2014). "Family Involvement Is Helpful and Harmful to Patients' Self-Care and Glycemic Control.", Patient Education and Counseling 97(3), p.p: 418-25.

12. Niccoli, Teresa, and Linda Partridge. (2012), "Ageing as a Risk Factor for Disease.", Current Biology 22(17), p.p: 741-52.

13. Pai, Chintamani, Ankush Bhaskar, and Vaibhav Rawoot. (2020). "Investigating the Dynamics of COVID-19 Pandemic in India under Lockdown.", Chaos, Solitons, and Fractals 138: 109988. 
14. "Public Poll: Emergency Care Concerns Amidst COVID-19."

https://www.emergencyphysicians.org/ article/COVID19/public-poll-emergency-careconcerns-amidst-COVID-19 (February 26, 2021).

15. Rodríguez-Leor, Oriol et al. (2020). "Impact of the COVID-19 Pandemic on Interventional Cardiology Activity in Spain." REC: interventional cardiology (English Edition): 4060.

16. Roy, Deblina et al. (2020), "Study of Knowledge, Attitude, Anxiety \& Perceived Mental Healthcare Need in Indian Population during COVID-19 Pandemic.”, Asian Journal of Psychiatry 51: 102083.

17. Wilhelm, Jess Alan, and Stéphane Helleringer. (2021), "Utilization of Non-Ebola Health Care Services during Ebola Outbreaks: A Systematic Review and Meta-Analysis." Journal of Global Health 9(1), https://www.ncbi.nlm.nih.gov/pmc/articles/PMC6 $344071 \%$.

18. World Health Organization. (February 11, 2021) "Maintaining Essential Health Services." https://www.who.int/emergencies/diseases/novelcoronavirus-2019/related-health-issues.

\section{TABLES AND FIGURES}

Table 1: Department wise missed appointments in frequency \& percentage

\begin{tabular}{|l|c|c|c|c|}
\hline \multirow{2}{*}{ Department } & \multicolumn{2}{c|}{$\begin{array}{c}\text { Total Attended } \\
\text { Appointments }\end{array}$} & \multicolumn{2}{c|}{$\begin{array}{c}\text { Samples from Missed } \\
\text { Appointments }\end{array}$} \\
\cline { 2 - 5 } & Frequency & $\mathbf{( \% )}$ & Frequency & $\mathbf{( \% )}$ \\
\hline Medical departments & 92752 & 51.3 & 87 & 67.7 \\
\hline Surgical departments & 44492 & 24.6 & 14 & 13.8 \\
\hline Paediatrics & 7418 & 4.1 & 10 & 7.7 \\
\hline Obstetrics \& Gynaecology & 16123 & 8.9 & 11 & 6.1 \\
\hline Emergency and others & 19965 & 11.04 & 8 & 4.6 \\
\hline Total & 180750 & 100 & 130 & 100 \\
\hline
\end{tabular}

Fig -1: System wise illness percentage reported in missed patient appointments

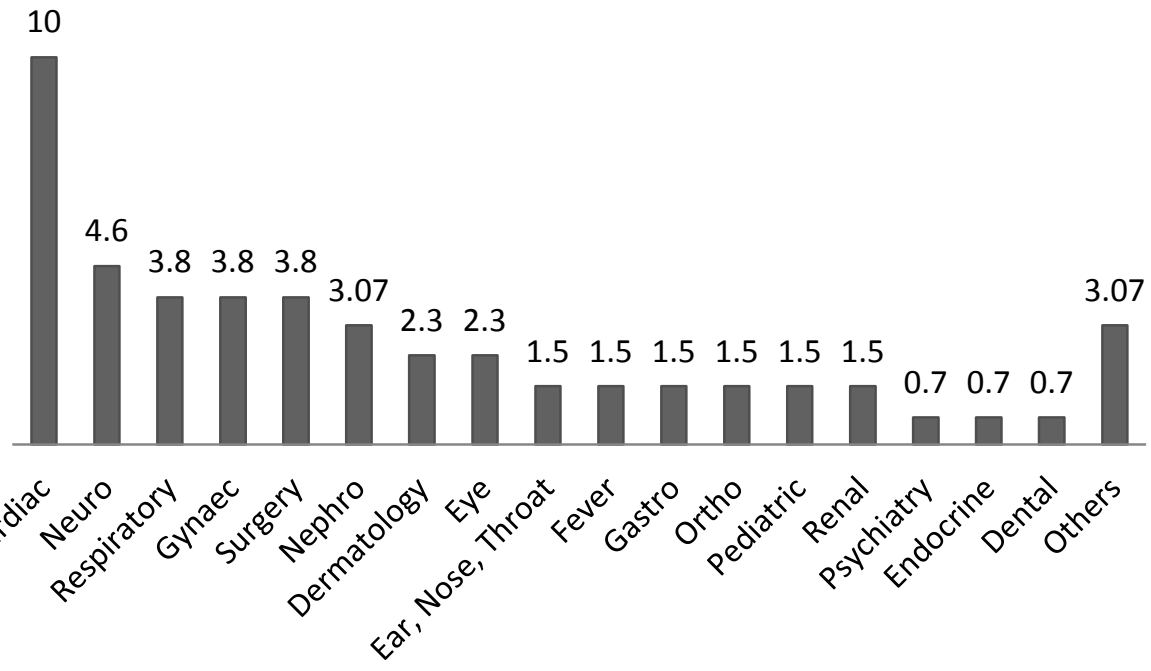


Figure 2: Factors lead the patients to missed appointments

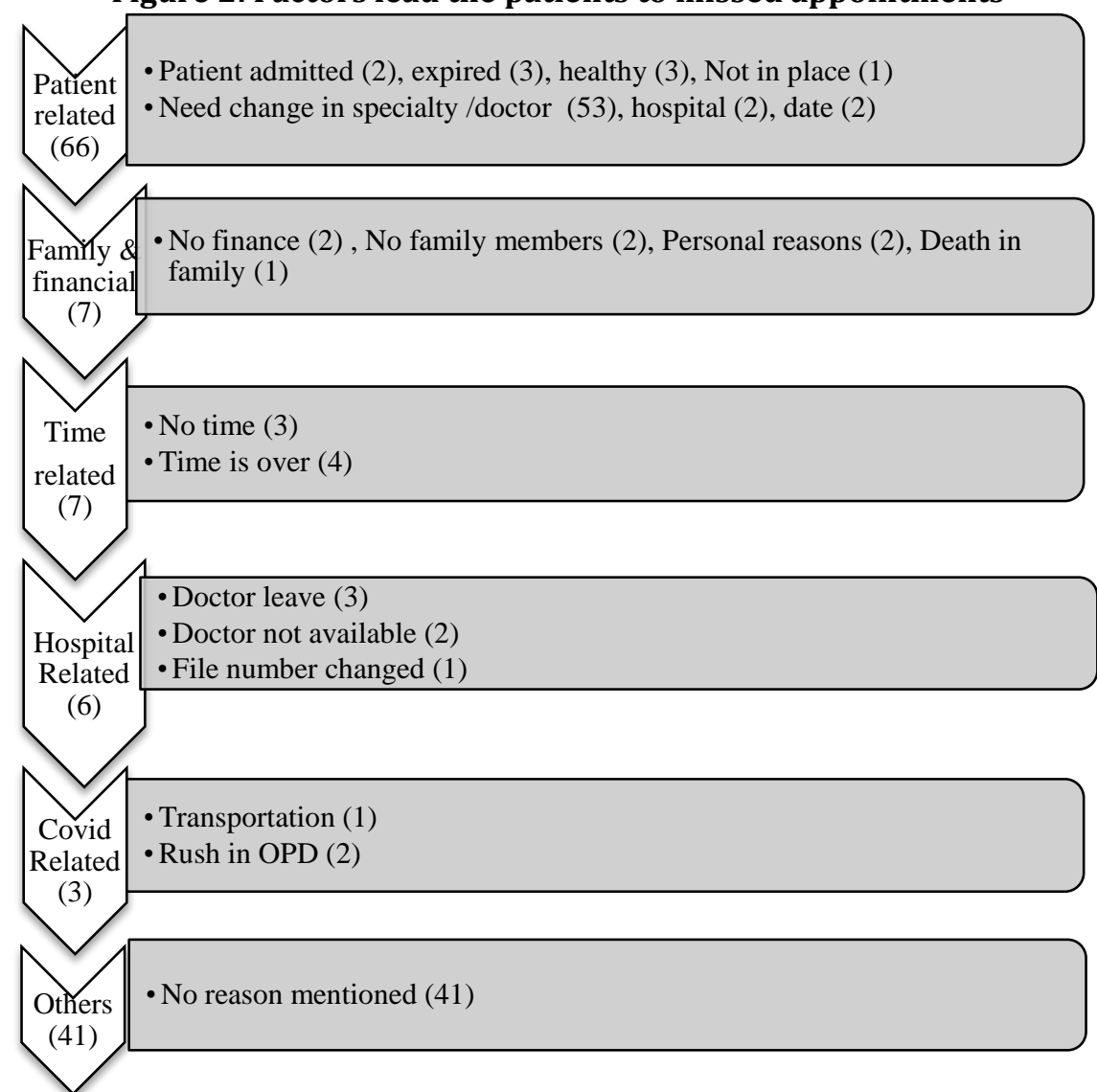

